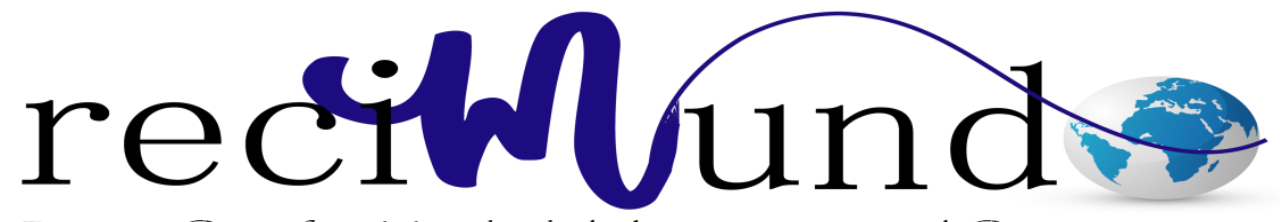

Revista Científica Mundo de la Investigación y el Conocimiento

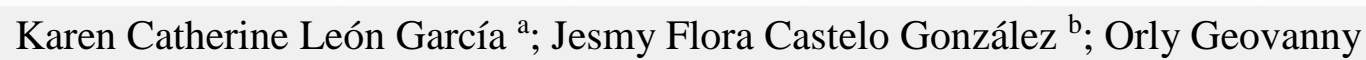
Huerta Chamorro ${ }^{\mathrm{c}}$

Estudio del nivel de participación de las PYMES del cantón Milagro en los procesos de contratación pública para captar nuevos negocios

Revista Científica Mundo de la Investigación y el Conocimiento. Vol. 2 núm., especial, mayo, ISSN: 2588-073X, 2018, pp. 269-283

DOI: 10.26820/recimundo/2.esp.2018.269-283

Editorial Saberes del Conocimiento

Recibido: 15/12/2017

Aceptado: 24/02/2018

a. Universidad Estatal de Milagro.

b. Universidad Estatal de Milagro.

c. Universidad Estatal de Milagro. 


\section{Estudio del nivel de participación de las PYMES del cantón Milagro en los procesos de contratación pública para captar nuevos negocios \\ Vol. 2, núm. Esp., (2018) \\ Karen Catherine León García; Jesmy Flora Castelo González; Orly Geovanny Huerta Chamorro}

\section{RESUMEN}

En la ciudad de Milagro desde la existencia de la Ley Orgánica de Contratación Pública, las Pymes registradas en el RUP han dejado pasar durante muchos años, la oportunidad de crecer y de expandirse a otros rincones de la provincia y del país, debido a que no han sabido aprovechar la gama de oportunidades que el Gobierno Central brinda a través de la contratación pública, a todo este importante sector productivo, es por esta razón que se ha considerado importante la realización del presente trabajo investigativo, el mismo que se ha constituido en una brillante oportunidad para indagar, buscar e investigar, sobre la relación e influencia directa que existe entre el nivel de suscripción de las Pymes de la ciudad de Milagro, con el nivel de desarrollo económico de esta ciudad, para lo cual se elaboraron las respectivas hipótesis para su posterior validación, mediante la información obtenida a través del proceso de encuesta, las mismas que estuvieron dirigidas a todas las Pymes acantonadas en este rincón de la provincia del Guayas. Cabe destacar que con el desarrollo de este estudio se ha incursionado en nuestra ciudad en una temática moderna y original, debido a que no se trata de los típicos temas que tienen abarrotadas las perchas de las bibliotecas, de las diferentes instituciones de educación media y superior. La parte medular de este estudio que es a su vez la más relevante, radica en la presentación clara y detallada de las causas que originan la poca participación de las Pymes, en el mundo moderno de las compras públicas, así como el planteamiento de estrategias innovadoras que permitan dar un giro de 360 grados, a esta panorámica local que se vive en la actualidad.

Palabras claves: Contratación pública, montos de adjudicación, tipo de procesos, nivel de participación. 


\title{
Estudio del nivel de participación de las PYMES del cantón Milagro en los procesos de contratación pública para captar nuevos negocios
} Vol. 2, núm. Esp., (2018)

Karen Catherine León García; Jesmy Flora Castelo González; Orly Geovanny Huerta Chamorro

\begin{abstract}
In the city of Milagro from the existence of the Law on Government Procurement, SMEs registered in the ORs have missed for many years, the opportunity to grow and expand to other parts of the province and the country, because they do not They have taken advantage of the range of opportunities that the Central Government provided through government procurement, all this important productive sector, is for this reason that it seemed important to carry out this research work, the same that has become a brilliant opportunity to investigate, search and investigate the relationship and direct influence between the level of participation of small and medium enterprises in the city of Milagro, with the level of economic development of this city, which were developed for the respective hypotheses for further validation using information obtained through the survey process, the same as they were aimed at all SMEs stationed in this corner of the province of Guayas. Notably with the development of this study it has ventured into our city into a modern and original theme, because it is not the typical issues that have crowded hangers libraries of different institutions of secondary and higher education. The core of this study is to turn the most relevant, is in the clear and detailed presentation of the causes of the low participation of SMEs in the modern world of public procurement, as well as the approach of innovative strategies that allow for a 360 degree panorama that this local live today.
\end{abstract}

Keywords: Public procurement, award amounts, type of process, level of participation. 


\section{Estudio del nivel de participación de las PYMES del cantón Milagro en los procesos de contratación pública para captar nuevos negocios \\ Vol. 2, núm. Esp., (2018) \\ Karen Catherine León García; Jesmy Flora Castelo González; Orly Geovanny Huerta Chamorro}

\section{Introducción.}

El Ecuador por ser un país que día a día avanza, el entorno de su jurisprudencia gubernamental es muy cambiante y evoluciona en función de las metas macros que los gobernantes de turno consideran pertinente dentro de su planificación a corto, mediano y largo plazo. Esto repercute en todo nivel, es decir, en las relaciones comerciales y diplomáticas a nivel internacional y en lo nacional a niveles de instituciones públicas, privadas e inclusive a nivel familiar. (Lima Fosado, 2007)

Muchas de estas leyes intentan una redistribución de las riquezas y creación de oportunidades de manera igualitaria e incluyente. Dentro de este contexto, las pymes ecuatorianas han tenido la oportunidad de participar y ofertar sus bienes y servicios a las instituciones estatales, en similitud de condiciones con las medianas y grandes empresas, es decir las pymes han dejado de ser un simple espectador desde los diferentes sectores económicos en los cuales se desempeñan, para convertirse en una pieza fundamental del engranaje del aparato productivo de este país. (Zapata Guerrero, 2004) (Góngora Biachi \& Madrid Guijarro, 2010)

Cabe señalar que estos cambios a nivel jurídico se han dado simultáneamente con el desarrollo tecnológico que el mundo vive diariamente, es decir, que el Gobierno Central en uso de sus facultades, ha desarrollado softwares que permiten realizar actividades licitas de compras a nivel estatal, y lo ha hecho por medio de la instauración del Sistema Oficial de Contratación del Estado (SOCE), una importante herramienta informática, la misma que fue creada e implementada por el Servicio Nacional de Contratación Pública (SERCOP) y a la cual se puede acceder a través de la página web www.compraspublicas.gob.ec. 


\section{Estudio del nivel de participación de las PYMES del cantón Milagro en los procesos de contratación pública para captar nuevos negocios}

Vol. 2, núm. Esp., (2018)

Karen Catherine León García; Jesmy Flora Castelo González; Orly Geovanny Huerta Chamorro

Actualmente esta herramienta virtual es el principal instrumento por medio del cual las Entidades estatales, realizan sus adquisiciones de bienes y servicios, así como la contratación de obras y consultorías especializadas en una determinada área del conocimiento. A partir del 04 de agosto del año 2008, el uso de este portal se volvió obligatorio tanto para compradores (empresas públicas), como para vendedores (personas naturales o jurídicas privadas), producto de ello, año a año se publican cientos de procesos precontractuales, que representan millones de dólares de desembolsos de dineros públicos. (Marulanda Echeverry, Giraldo García, \& López Trujillo, 2012)

Uno de los propósitos del Gobierno Central es suministrar igualdad de oportunidades de negocios para las pequeñas, medianas y grandes empresas, se pensaría entonces, que los recursos generados por las transacciones se distribuyen equitativamente entre todos los oferentes, pero resulta que estos dineros están siendo captados en su gran mayoría por las medianas y grandes compañías, tanto nacionales como extranjeras que tienen su domicilio fiscal en el Ecuador, gracias a que poseen un fuerte capital de trabajo mientras que las pequeñas y microempresas tienen que hacer un esfuerzo gigante para poder competir por esos negocios, debido a que por su naturaleza no disponen de un capital que permita maniobrar financieramente ante cualquier exigencia de los demandantes de bienes y servicios, ni tampoco disponen de un fácil acceso a créditos que se encuentran disponibles en las diferentes instituciones del sistema financiero nacional. En otras palabras, lo ideal debería ser, que esta riqueza ecuatoriana, sea distribuida equitativamente entre todos los actores de la economía nacional, para que puedan disfrutar de este beneficio de forma justa. (Rincón, 2008) 


\section{Estudio del nivel de participación de las PYMES del cantón Milagro en los procesos de contratación pública para captar nuevos negocios}

Vol. 2, núm. Esp., (2018)

Karen Catherine León García; Jesmy Flora Castelo González; Orly Geovanny Huerta Chamorro

El objetivo de la presente investigación es identificar el nivel de participación que tienen las Pymes del Cantón Milagro en los procesos de Contratación Pública, mediante un estudio detallado a ésta industria.

\section{Metodología.}

Tipo y diseño de investigación

Las características con las que cuenta esta investigación son las siguientes:

Según su objetivo gnoseológico

Descriptiva: Porque se va a identificar todas las características por las que las Pymes no aprovechan las oportunidades de negocios que se encuentran diariamente, en el portal de contratación pública del SERCOP.

Explicativas: Porque se realizará una explicación de cada una de las hipótesis que se han planteado en el presente estudio investigativo.

Transversal o transaccional: Porque se va a trabajar con información actualizada y real tomada directamente de los representantes legales de las Pymes, mediante el uso de una encuesta clara y precisa. 


\section{Estudio del nivel de participación de las PYMES del cantón Milagro en los procesos de contratación pública para captar nuevos negocios}

Vol. 2, núm. Esp., (2018)

Karen Catherine León García; Jesmy Flora Castelo González; Orly Geovanny Huerta Chamorro Según contexto:

De campo: Por medio de este trabajo de investigación, se determinará con exactitud cuáles son las diferentes causas y motivos que inciden en la no participación de las Pymes, en los procesos de compras públicas, dato obtenido directamente de los sectores empresariales estudiados.

La población y la muestra

\section{Características de la población}

La población para el presente estudio estará conformado por todas las Pymes de la ciudad de Milagro que están inscritas en el Servicio Nacional de Contratación Pública. Esta información será obtenida de la base de datos de proveedores que posee el Gobierno Autónomo Descentralizado del cantón Milagro y el SERCOP, para de esta manera conocer con exactitud cuáles son las Pymes que forman parte de esta población.

\section{Delimitación de la población}

La población con la que se trabajará en la presente investigación es finita, debido a que se dispone con exactitud el número de Pymes del Cantón Milagro que poseen un Registro Único de Proveedores, el mismo que alcanza la cantidad total de 68 Pymes.

Tipo de la muestra 


\section{Estudio del nivel de participación de las PYMES del cantón Milagro en los procesos de contratación pública para captar nuevos negocios}

Vol. 2, núm. Esp., (2018)

Karen Catherine León García; Jesmy Flora Castelo González; Orly Geovanny Huerta Chamorro

El tipo de muestra de esta investigación es no probabilística, por cuanto las personas seleccionadas para aplicarles el instrumento de la encuesta, son los Representantes Legales de las Pymes de la ciudad de Milagro.

\section{Tamaño de muestra}

Para el presente estudio investigativo se considerará como tamaño de la muestra al total de la población, en vista de que esta posee un tamaño que permite aplicar a todos las Pymes, el respectivo instrumento diseñado para tal efecto. En este caso el tamaño exacto de la muestra estará compuesto por 68 Pymes milagreñas, quienes son los inscritos en el R.U.P. con domicilio en el cantón Milagro, en otras palabras se decidió estudiar a toda la población por tratarse de una cantidad pequeña y accesible, que no demandará mucho tiempo en la aplicación del instrumento que posee el cuestionario de preguntas, ni tampoco generará grandes gastos por concepto de movilización o de impresión.

\section{Proceso de Selección}

Para este punto no se utilizará un proceso de selección en especial, por cuanto todos los elementos que forman parte de la población estudiada serán encuestados.

\section{Los métodos y las técnicas}

\section{Métodos teóricos}

Analítico-Sintético: Se utilizará este método por cuanto se va a analizar detenidamente todos los factores que ocasionan la existencia de esta problemática. 


\section{Estudio del nivel de participación de las PYMES del cantón Milagro en los procesos de contratación pública para captar nuevos negocios}

Vol. 2, núm. Esp., (2018)

Karen Catherine León García; Jesmy Flora Castelo González; Orly Geovanny Huerta Chamorro Métodos empíricos

- Fundamentales: Se utilizará la observación en la aplicación de este método

- Complementarios: Se utilizará la encuesta en la aplicación de este método

Técnicas e instrumentos

La encuesta.- Esta instrumento servirá para obtener información relevante en la obtención de la solución a la problemática objeto de estudio, el mismo que estará compuesto por un cuestionario de diez preguntas, es decir servirá para comprobar y verificar la hipótesis planteada en la propuesta.

Procesamiento estadístico de la información

El procesamiento estadístico de la información que se va a utilizar, será realizado a través del uso del programa Excel, en el cual se creará una base de datos detallada que servirá para tabular todos los datos obtenidos y de esta forma presentar estos resultados, a través de gráficos tipo pastel, barras o líneas, con la finalidad de realizar el análisis e interpretación individual de cada uno de estos gráficos.

\section{Resultados.}

Una vez que se ha finalizado con el proceso de aplicación y tabulación de encuestas a las Pymes de la ciudad de Milagro, se procede a presentar un resumen de la información obtenida.

Al realizar una pregunta para conocer el número de concursos en los cuales participan anualmente las Pymes, a través del portal del SERCOP se observa que su participación en estos 


\section{Estudio del nivel de participación de las PYMES del cantón Milagro en los procesos de contratación pública para captar nuevos negocios}

Vol. 2, núm. Esp., (2018)

Karen Catherine León García; Jesmy Flora Castelo González; Orly Geovanny Huerta Chamorro

concursos es muy baja, es decir poco o nada les interesa participar en estos importantes y lucrativos

eventos de negocios, publicados por las empresas estatales ecuatorianas, lo cual se sustenta en el 0,24\% de participación que tienen en relación al 100\% de procesos publicados. Esta situación es perjudicial para este importante sector de la economía nacional, puesto que al no saber aprovechar estas oportunidades, están perdiendo importantes recursos económicos que les servirían para su crecimiento financiero, lo que a su vez se traduciría en la expansión de la empresa ya sea a nivel local o nacional, lo que significaría crear nuevas plazas de trabajo, ofrecer una mejor remuneración a su personal, mejorar los márgenes de ganancia, etc.

Al realizar otra interrogante para conocer el nivel de facturación proveniente de los negocios realizados con el Estado ecuatoriano, se puede ver que los dineros captados del sector público son escasos, considerando los millones de dólares que son adjudicados mes a mes a través del sistema nacional de contratación pública, lo cual se refleja en apenas el 0,003\% del total facturado al Estado ecuatoriano a nivel nacional. Esta situación es producto de la escaza participación que tienen las Pymes en los concursos publicados día a día, a través de los diferentes procesos estipulados en la LOSNCP y su Reglamento.

Al efectuar una pregunta con la finalidad de conocer el número de procesos que le han sido adjudicados a las Pymes en el presente año 2015, podemos observar que la cantidad es muy baja, es decir hablando a nivel porcentual este valor no llega ni siquiera al $1 \%$ de la masa total de procesos que son adjudicados en el portal informático del SERCOP, pues a duras penas es del 0,12\% con lo cual se ratifica que las Pymes de esta localidad tienen una participación baja y una 


\section{Estudio del nivel de participación de las PYMES del cantón Milagro en los procesos de contratación pública para captar nuevos negocios}

Vol. 2, núm. Esp., (2018)

Karen Catherine León García; Jesmy Flora Castelo González; Orly Geovanny Huerta Chamorro presencia casi nula en los concursos creados por las diferentes Entidades y organismos del sector público ecuatoriano.

Al plantear una interrogante con el ánimo de saber cuáles son los factores por los cuales las Pymes milagreñas no están inscritas en el Registro Único de Proveedores, se obtuvo como principal motivo la falta de conocimiento en contratación pública, con un alto porcentaje del $50 \%$ constituyéndose de esta manera en la principal causa de esta situación adversa. En segundo lugar se conoce que la desconfianza en la gestión de las Entidades públicas, es la causante de esta problemática, lo cual demuestra que en la actualidad sigue habiendo un alto porcentaje de empresarios que ven con malos ojos a las Empresas del Estado ecuatoriano, el mismo que alcanza el $19 \%$.

Con un porcentaje del $15 \%$ los consultados respondieron que el poco Capital de trabajo es el motivo por el cual no se inscriben en el RUP, mientras que un $10 \%$ mencionó que el hecho de tener que cumplir con un excesivo número de requisitos al momento de participar en un proceso, es lo que los desmotiva a inscribirse en el SERCOP y finalmente un 6\% de los encuestados menciona que el porcentaje mínimo de ganancia en los procesos de contratación pública, es la causa principal por la cual no piensan inscribirse en el RUP.

Al efectuar una pregunta con la intención de conocer cuáles son las razones por las que las Pymes no aceptan las invitaciones, que reciben mensualmente a través del portal del SERCOP, se encontró como el principal factor el desconocimiento en la forma de armar las carpetas, causal que alcanzó el 35\% es decir esto está íntimamente ligado con el desconocimiento en materia de contratación pública, por otra parte con un 26\% se ubicó como la segunda causa de esta situación 


\section{Estudio del nivel de participación de las PYMES del cantón Milagro en los procesos de contratación pública para captar nuevos negocios}

Vol. 2, núm. Esp., (2018)

Karen Catherine León García; Jesmy Flora Castelo González; Orly Geovanny Huerta Chamorro

al hecho de que las Pymes no revisan a tiempo las invitaciones que reciben, lo que se traduce en un descuido grave por parte de ellos, debido a que actualmente estas invitaciones son recibidas tanto por el correo personal como a través de la página web del SERCOP.

Otro de los factores que influye en esta situación es la desconfianza existen en los diferentes procesos por parte de las Pymes, porcentaje que alcanzó el 19\%, mientras que con un 12\% se ubicó como justificación el hecho de que las bases están direccionadas a grandes empresas y finalmente en último lugar con un $7 \%$ se estableció como motivo a la falta de anticipo en los procesos de contratación pública.

En el momento de realizar una pregunta con la finalidad de saber cuál es el motivo por el cual las Pymes, que participan en los procesos de contratación pública no logran ser adjudicadas, se encontró como el principal motivo con un 36\% al hecho de que las carpetas están realizadas de forma incompleta, es decir estas ofertas técnicas no presentan la totalidad de los documentos y requisitos solicitados en los pliegos, por las empresas pertenecientes al sector público ecuatoriano; en el segundo lugar con un 29\% se ubicó como causal a las fallas en la realización de la oferta, es decir a pesar de que las carpetas contienen toda la documentación que se pide en un concurso, estas están mal llenadas, ya sea en su parte técnica, en nombres, códigos de proceso, cantidades, etc.

Como un tercer factor con un $18 \%$ los encuestados dijeron que era por entregar fuera de tiempo las ofertas técnicas, es decir a pesar de presentar todos los documentos y requisitos solicitados en los pliegos, la oferta en sobre cerrado es entregada extemporáneamente, lo cual según la LOSNCP es un motivo directo para descalificar una carpeta, sin opción a reclamo alguno por parte del participante. Como un cuarto motivo se estableció el no subir la oferta durante la 


\section{Estudio del nivel de participación de las PYMES del cantón Milagro en los procesos de contratación pública para captar nuevos negocios}

Vol. 2, núm. Esp., (2018)

Karen Catherine León García; Jesmy Flora Castelo González; Orly Geovanny Huerta Chamorro puja, en otras palabras esto ocurre por fallas en el sistema informático del SERCOP o debido a que el proveedor concursante es inhabilitado por el SERCOP, por mantener deudas con el Estado, ya sea con empresas como el Servicio de Rentas Internas, el Instituto Ecuatoriano de Seguridad Social, el Banco de Fomento, la Corporación Financiera Nacional, etc., o simplemente porque el participante se olvidó subir en el portal de compras públicas el valor inicial para la puja electrónica.

Finalmente como la última causa de esta situación se ubicó la creencia de algunas Pymes, de que en un concurso ya existían ganadores previos, esto producto de información privilegiada recibida o por el tipo de requisitos y documentos que se solicitan en los pliegos precontractuales, en la sección destinada para las especificaciones técnicas y términos de referencia.

\section{Conclusiones.}

Se ha demostrado mediante el presente trabajo que el nivel de participación de las Pymes de Milagro, en los procesos de compras públicas del país es sumamente bajo en relación a la cantidad de procesos, que publican anualmente las Entidades del sector público. La participación activa de las Pymes en este tipo de procesos no alcanza ni siquiera el $1 \%$.

El número de Pymes de la ciudad de Milagro que poseen Registro Único de Proveedores es de 68 .

El monto facturado al Estado por parte de las Pymes de Milagro durante el año 2014, fue de $\$ 253,000.00$ el cual equivale al 0,003\% del total vendido al sector público.

El número de procesos de contratación pública adjudicados a las Pymes de Milagro es de 116. Adicional a esto se estableció que de las Pymes milagreñas que poseen RUP tan sólo el 22\% 


\section{Estudio del nivel de participación de las PYMES del cantón Milagro en los procesos de contratación pública para captar nuevos negocios}

Vol. 2, núm. Esp., (2018)

Karen Catherine León García; Jesmy Flora Castelo González; Orly Geovanny Huerta Chamorro

de ella participa en los concursos de compras públicas, que son administrados a nivel nacional por el Servicio Nacional de Contratación Pública.

Finalizado el proceso de encuesta se pudo observar que las principales causas por las cuales las Pymes no participan en los concursos del portal del SERCOP, son la falta de conocimientos en contratación pública y la desconfianza que existe hacia la gestión que se desarrolla en el interior de las empresas y organismos pertenecientes al sector público ecuatoriano.

El presente trabajo de investigación puede ser replicado y aprovechado por el GAD Municipal del cantón Milagro, para que este a su vez ayude a incentivar a las Pymes de esta localidad y así se decidan a ser parte activa en el mundo de los negocios con el Estado ecuatoriano.

Se concluye finalmente que las Pymes de la ciudad de Milagro están perdiendo la oportunidad de captar altos recursos económicos, provenientes de las empresas públicas, lo cual afecta directamente su crecimiento y le merma posibilidades de expandirse a otras ciudades del territorio nacional.

Se ratificó la totalidad de las hipótesis planteadas, una vez tabulados los resultados que se obtuvieron de parte de los representantes legales de las Pymes encuestadas.

\section{Bibliografía.}

Góngora Biachi, G., \& Madrid Guijarro, A. (2010). El apoyo a la innovación de la PyME en México. Un estudio exploratorio. Investigación y Ciencia, 18(47), 21-30.

Lima Fosado, R. (2007). Información financiera en las PYMES. Revista del Centro de Investigación, 7(27), 67-75. 


\section{Estudio del nivel de participación de las PYMES del cantón Milagro en los procesos de contratación pública para captar nuevos negocios}

Vol. 2, núm. Esp., (2018)

Karen Catherine León García; Jesmy Flora Castelo González; Orly Geovanny Huerta Chamorro

Marulanda Echeverry, C., Giraldo García, J., \& López Trujillo, M. (2012). Herramienta para medición de la gestión del conocimiento en las PYMES de Colombia. Revista Virtual Universidad Católica del Norte, 35(Feb), 83-104.

Rincón, M. (2008). Los entornos virtuales como herramientas de asesoría académica en la modalidad a distancia. Revista Virtual Universidad Católica del Norte, 25(Sep).

Zapata Guerrero, E. (2004). Las PyMES y su problemática empresarial. Análisis de casos. Revista Escuela de Administración de Negocios, 52(Sep), 119-135. 